\title{
A Study of Smartphone Based Disaster Information Reporting System under Disaster Environment
}

\author{
Yin Baoquan $^{1,2}$, Sun Ruizhi ${ }^{1}$ and Yang Hongjun ${ }^{1}$ \\ 1. Key Laboratory of Agricultural Information Acquisition Technology, Ministry \\ of Agriculture of the People's Republic of China, Beijing, P.R. China \\ 2. Yantai Academy of China Agricultural University, Yantai, Shandong, P.R. \\ China \\ yinbaoquan@126.com,sunrz_cn@sina.com.cn
}

\begin{abstract}
With the development of technology and communication network, smartphones are increasingly used in emergency platforms. Under disaster environment, however, the information reporting system may confronted with poor communication conditions or low phone battery. In this study we first defined two parameters: the communication signal and the battery status. Based on the definitions, we proposed an algorithm for smartphone based reporting system under disaster environment. With the guarantee that disasters can be reported promptly and accurately and based on the analysis of smartphone power consumption, the algorithm provides power saving mechanisms to obviously increase the system working time.

At last, a smartphone based disaster information reporting system (MDIRS) is designed and applied in disaster environment.
\end{abstract}

Keywords: disaster, information reporting, smartphone, power consumption

\section{Introduction}

Natural disasters have caused huge damages and losses to both human lives and belongs. The effective and accurate reporting of disasters is critical in in disaster emergency management. With the development of communication technology network, smartphones has been increasingly used in emergency platforms, which has been extensively discussed in literatures [1-4]. These studies focused on the scenarios that both smartphones and cellular network are readily available. However, under natural disasters, such as earthquakes, floods and snow freezing disasters, there might be extensive damage to both power supply system and communication infrastructure. As a result, the communication may be interrupted. Furthermore, the high power consumption of smartphones is a major obstacle in applying smartphones to disaster information reporting. Hence, the study of disaster information reporting system with smartphones under disaster environment is critical.

\section{Related Work}

Generally, the process of disaster information reporting with smartphones is as below. If a natural disaster happens, the disaster information, including time, location, classification, degree, trend of disaster, etc., need be filled in the disaster information reporting system (DIRS). Then, the disaster information is sent to the emergency management center through the information reporting system installed on a smartphone. Among them, the geometry location of the disaster is provided by the smartphone's location based services. Reporting category can be classified into the primary report and the subsequent report. The primary report is defined as the 
report in when the disaster is first discovered. Subsequent report is a report following the disaster evolvement after the first report for continuous information updating, which includes the current disaster status and the development trend. For a straightforward description in helping the management center respond to the disaster, the report may include sounds, images and videos besides text. The previous process is based on the fact that both the smartphone and communication network work well. In a natural disaster, however, the following scenarios may happen:

(1) Low battery. Smartphones are offering functionalities such as GPS, Wi-Fi access, and multimedia services. Frequent networking requests, GPS positioning, and frequent CPU task processing have high demands to power which decreases the battery duration. Once a mobile phone's battery runs out, no further functions including disaster reporting can be provided. Hence, it is very vital to leave sufficient battery for rescue and relief during a disaster.

(2) Erratic or interrupted communication. Natural disasters may destroy the power supply and communication infrastructure which results in unstable, weak or interrupted communication signals. Mobile phones will drain extra battery power to search constantly for a signal when the signal is weak.

Battery capacity is severely restricted by the size and weight of a smartphone. This implies that energy efficiency of smartphones is very important to their usability. Hence, optimal management of power consumption is critical. Dynamic power management (DPM) is a design methodology that dynamically reconfigures a system to minimize the unnecessary power consumption of a smartphone by selectively turning off (or reducing the performance of) system services when they are idle [5]. Some studies showed that wireless data transmission and location-based services consume much of a smartphone's battery [6-8]. Shih et al. proposed an energy saving strategy, called wake-on-wireless, by which the wireless network adaptor of the smartphone is shut down when it is not in use and powered on only when an incoming call is received [9]. Compared with Wi-Fi, cellular network requires much lower power to stay connected but incurs much higher energy per MB transferred. Therefore, Rahmati and Zhong suggested to leverage the complementary strength of $\mathrm{Wi}-\mathrm{Fi}$ and cellular network to improve the energy efficiency by choosing the wireless interface for data transfer based on the network condition estimation [10].

There are two primary positioning technologies currently in use: the satellite positioning and the network positioning. The satellite positioning technology mainly includes global positioning system (GPS), Beidou navigation satellite system (BDS) and so on. The network positioning technology uses the locations of some nearest base stations (such as GSM, CDMA, etc.) or locations of Wi-Fi base stations to calculate mobile phone's position. The positioning accuracy usually relies on the number and distributions of the base stations in the surrounding area [11]. The satellite positioning service is generally more accurate, but slower in positioning and requiring higher battery drain than the network positioning service. More and more studies are focusing on how to reduce power consumption when the positioning resolution can meet requirements. The following solutions are usually adopted: reducing the positioning frequency, choosing power-saving positioning services, and sending less data and less often, etc. [12].

Based on the consideration of both factors of mobile phones' battery and communication state, we propose in this study a strategy of information reporting with smartphones under disaster environment. 


\section{Algorithm of Information Reporting Under Disaster Environment}

The following strategy is the basic idea of an information reporting system under disaster environment: firstly make sure the basic disaster information being sent in time, and then use power-saving technologies to extend the battery lifetime of mobile phones. As mentioned above, the power-saving technology of smartphones mainly include two aspects: wireless data transfer technology and positioning technology. There is usually no Wi-Fi access at the site of a nature disaster. In the rare case that Wi-Fi signals are available, the cell phone can then be readily charged. Hence, only the positioning technology is considered in modeling the system.

\subsection{System Modeling}

In order to send disaster information in time, a disaster information message is divided into two parts: basic information and multimedia information. Compared to the basic information, the multimedia information has huge data and is less important. We define $T$ as the type of information message. When $T=1$, the message is a basic information message. When $T=0$, it is a multimedia information message. We also define $S_{G}$ as the communication status and $S_{p}$ as the battery status. The sending mode, $M$, for judging how to send the message is a function of $T, \mathrm{~S}_{p}$ and $S_{G}$,

$$
M=f\left(S_{p}, S_{G}, T\right) .
$$

In poor cellular signal coverage area, the mobile phone requires higher transmit power (TX) in order to keep connection with base stations which results in higher power consumption and hence lower battery lifetime. Battery lifetime relies on not only the battery status $S_{p}$ but also the communication status $S_{G}$. Further, different smartphone models and different mobile carriers affect battery lifetime differently. In other words, the function in formula (1) is complex and nonlinear. To approximate $\mathrm{M}$ in practice, we simplify formula (1) as below based on experiences.

We consider the battery status $S_{p}$ at first. Generally, a smartphone needs to be recharged while the battery is less than $5-10 \%$. Otherwise, it will turn off automatically. In order to have the basic function of a mobile phone available in emergencies, the minimum value for battery is set to $10 \%$. The definition of battery status $S_{p}$ is shown in the Table 1 .

Table 1. Definition of Battery Status

\begin{tabular}{cc}
\hline Battery & Battery status \\
\hline $100 \% \sim 30 \%$ & 2 (sufficient) \\
$30 \% \sim 10 \%$ & 1 (low) \\
$0 \sim 10 \%$ & 0 (critically low) \\
\hline
\end{tabular}

The values in Table 1 are the default values provided by the system, which can be modified depending on the smartphone's specific state.

We then consider the communication status $S_{G}$. According to the signal strength of a mobile phone, its communication status is defined in Table 2. In addition, we define $N_{\max }$ as the maximum times that a message can be repeatedly sent. The communication is considered in an interrupted state if the number of failed sending attempts exceeds $N_{\max }$. In this case, $S_{G}$ is set to zero. 
Table 2. Definition of Communication Status

\begin{tabular}{cc}
\hline Signal strength $/ \mathrm{dBm}$ & Communication status \\
\hline$>-90$ & 2 (strong) \\
\hline$-90 \sim-110$ & 1 (weak) \\
\hline$<-110$ & 0 (interrupted) \\
\hline
\end{tabular}

The definition of sending mode $M$ is shown in Table 3 .

Table 3. Definition of Sending Mode

\begin{tabular}{cccc}
\hline Battery status & Communication status & Information type & $\begin{array}{c}\text { Sending } \\
\text { mode }\end{array}$ \\
\hline \multirow{3}{*}{2 (sufficient) } & 2 (strong) & $\times$ & 2 \\
\cline { 2 - 4 } & 1 (weak) & 1 & 2 \\
\cline { 2 - 4 } & 0 (interrupted) & 0 & 1 \\
\cline { 2 - 4 } 1 (low) & 2 (strong) & 1 & 1 \\
\cline { 2 - 4 } & 1 (weak) & 0 & 0 \\
\cline { 2 - 4 } & 0 (interrupted) & 0 & 0 \\
\hline 0 (critical low) & $\times$ & $\times$ & 0 \\
\hline
\end{tabular}

In the table, sending mode "2" represents that this message can be sent now; "1" represents that the system cannot sent the message but will automatically detect communication status at regular intervals; " 0 " represents that the system cannot sent the message and reminds the user that the program will automatically close in a short time.

The sending mode $\mathrm{M}$ can be calculated by the formula below,

$$
M\left(S_{p}, S_{G}, T\right)=\left\{\begin{array}{cc}
S_{p} \cdot S_{G} \cdot T & , S_{p} \leq 1 \\
\operatorname{sgn}\left[\left(S_{p}-1\right) \cdot S_{G} \cdot T-1\right] & , S_{p}>1
\end{array}\right.
$$

\subsection{Algorithms for Information Reporting System under Disaster Environment}

As mentioned above, there are two positioning technologies used by smartphones: GPS technology and network positioning technology. The locating accuracy of network positioning technology is lower than the GPS technology. Meanwhile, the interrupted communication or destroyed base stations by a natural disaster will further affect the location accuracy of network positioning technology. Hence, when the smartphone's battery is sufficient $\left(S_{p}=2\right)$ and the signal strength is weak or interrupted $\left(S_{G}<2\right)$, the GPS technology is preferred; when the battery is weak and signal strength is strong, the network technology is preferred. In other cases, the system will turn off the positioning services of the smartphone and remind the use to enter the disaster location data manually.

A GETPOSITION algorithm is designed to obtain disaster position based on the communication status and the battery status.
Algorithm 1. GETPOSITION
1. StartGps();
2. if $\left(\left(S_{\mathrm{p}}=2\right)\right.$ OR $\left(\mathrm{S}_{\mathrm{G}}<2\right.$ AND $\left.\left.\mathrm{S}_{\mathrm{p}}>0\right)\right)$ then
3. Location loc $=$ getLastKnownLocation(GPS_PROVIDER);
4. else if $\left(S_{p}=1\right.$ AND $\left.S_{G}=2\right)$ then
5. Location loc $=$ getLastKnownLocation(NETWORK_PROVIDER); 
6. else

7. closeGps(); //close GPS and enter position in a dialog box manually

8. end if

A list of messages are created based on their priority when several disaster information messages need be sent. Among them, the priority of a basic message is higher than a multimedia message. Because the duration of sending a basic message is usually short, a new higher priority message will not interrupt the sending process. However, in the process of sending a multimedia message, a new higher priority message will interrupt the process and send first. If the communication is interrupted while sending, the messages will be saved automatically. The system will start a timer to detect the network signal at a regular basis and send the messages automatically as soon as the communication is restored. In addition, if the value of $M$ is changed due to the changes from either $S_{p}$ or $S_{G}$ while sending messages, the sending mode for basic messages will retain. However, the sending mode of multimedia messages will be updated to the current value of $M$.

A GETMODE algorithm is designed for getting the current sending mode:

\section{Algorithm 2. GETMODE}

1. $\mathrm{S}_{\mathrm{p}}=$ GetPowerLevel( $)$;

//obtain the current battery

2. $\mathrm{S}_{\mathrm{G}}=$ GetSignalStrength () ;

3. if $S_{\mathrm{p}}>1$ then

4. $\mathrm{M}=\operatorname{sgn}\left[\left(\mathrm{S}_{\mathrm{p}}-1\right) * \mathrm{~S}_{\mathrm{G}} * \mathrm{~T}-1\right]$;

5. else

6. $\quad \mathrm{M}=\mathrm{S}_{\mathrm{p}} * \mathrm{~S}_{\mathrm{G}} * \mathrm{~T}$

7. end if

8. return $\mathrm{M}$;

A SENDREPORT algorithm is designed for sending information reporting messages:

\section{Algorithm 3. SENDREPORT}

1. nFailNumber $=0 ; \quad / /$ the number of consecutive failure to send

2. $\operatorname{mode}=$ GetMode () ; //call GETMODE

3. GetPostion(); //call GETPOSITION

4. CreateMsgList(); //create the list of messages according to the priority

5. if mode $=1$ then

6. while (SendMessage ()$==$ FALSE) $\{$ //send message according to the priority

7. $\quad$ nFailNumber++;

8. $\quad$ if ( nFailNumber $>\mathrm{N}_{\max }$ )

9. $\quad \mathrm{S}_{\mathrm{G}}=0$, go to step 1;

10. $\quad$ nFailNumber $=0$;

11. else if mode $=2$ then

12. SendReport(); //start timer and call SENDREPORT

13. else

14. PromptMessage(); //bring up a dialog box to remind the user

15. endif

\section{System Implementation}

Based on the algorithms above, we have implemented a smartphone based disaster information reporting system (called MDIRS) that can realize the efficient 
and accurate information reporting under disaster environments. The system is developed using Visual Studio .NET. The mobile operating system is Windows Mobile 6.5. The system provides functions such as disaster information primary reporting, subsequent reporting and express reporting. Here, express reporting is a simplified version of primary reporting. It can send report information more quickly by reducing input data. All data will be temporarily saved in the smartphone when communication is interrupted. Message will be send out when communication is restored. In addition, the system provides a function for reviewing the list of messages. The user can delete a message which does not need to be sent.

Figure 1 shows the primary reporting interface of the MIDRS system, where user can input the data for primary report. Since it is not the focus of this paper, the details of the implementation are not introduced.

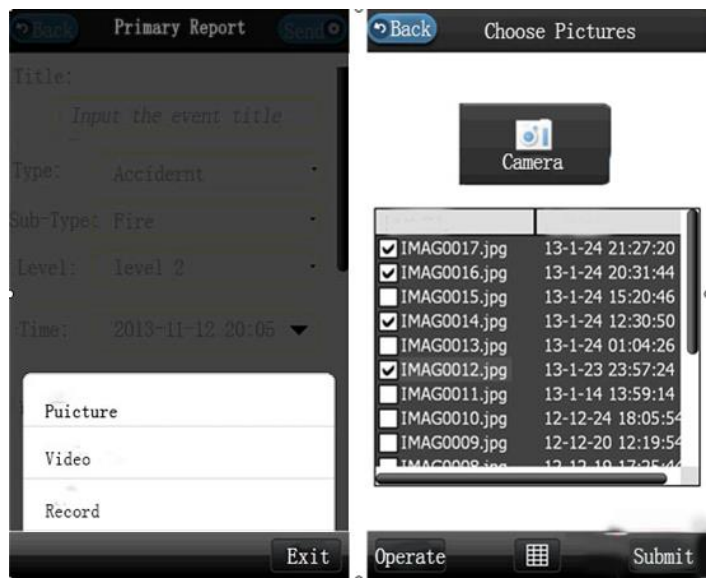

\section{Figure 1. The Main Interface of Primary Report}

\section{Conclusion}

The portability of smartphones and the rapid development of communication networks have improved the effectiveness and accuracy of disaster information reporting system. The functions of smartphones, such as taking photos, capturing videos and GPS positioning, have enhanced disaster information reports and facilitated decision making processes. These functions rely on the availability of smartphones and communication network. In the paper, based on the analysis of extreme conditions under disaster environment, we introduced two parameters, the communication status and battery status, to judge how to send disaster information report. This method not only ensures the basic principles of disaster information reporting but also adopts energy-saving measures to extend the lifetime of smartphone's battery and the availability of the reporting system.

\section{Acknowledgements}

This work is supported by the National Key Technology R\&D Program of China (2012BAH26B01), National Science and Technology Major Project of China (2012ZX01039-003 and 2012ZX01039-004-01-3). 


\section{References}

[1] S. Q. He and T. Wang, "Discussion on technique for earthquake rapid report and search and rescue in disaster area based on mobile phone positioning services, South China Journal of Seismology, vol. 32, no. 4, (2012), pp. 67-74.

[2] Y. G. Wu and L. He, "Application of Mobile GIS in Forest Fire Emergency Management System", Forest inventory and planning, vol. 35, no. 3, (2010), pp. 15-19.

[3] S. H. Seop, M. G. Young and J. D. Hoon, "A study on the development of disaster information reporting and status transmission system based on smart phone", ICT Convergence (ICTC), International Conference, IEEE, (2011), pp. 722-726.

[4] J. Q. Ma, M. G. Zhou, Y. F. Li, Y. Guo, X. M. Su, X. P. Qi and H. Ge, "Design and application of the emergency response mobile phone - based information system for infectious disease reporting in the Wenchuan earthquake zone", Journal of Evidence - Based Medicine, vol. 2, no. 2, (2009), pp. 115-121.

[5] L. Benini, A. Bogliolo and G. De Micheli, "A survey of design techniques for system-level dynamic power management”, Very Large Scale Integration (VLSI) Systems, IEEE Transactions, vol. 8, no. 3, (2000), pp. 299-316.

[6] M. Kjaergaard, "Minimizing the power consumption of location-based services on mobile phones", IEEE Pervasive Computing, vol. 8, no. 4, (2010).

[7] L. B. P. Consumption, "Location-based services on mobile phones: Minimizing power consumption", (2012).

[8] A. Carroll and G. Heiser, "An analysis of power consumption in a smartphone", Proceedings of the USENIX conference on USENIX annual technical conference, (2010), pp. 21-21.

[9] E. Shih, P. Bahl and M. J. Sinclair, "Wake on wireless: an event driven energy saving strategy for battery operated devices", Proceedings of the 8th annual international conference on Mobile computing and networking, ACM, (2002), pp. 160-171.

[10] A. Rahmati and L. Zhong, "Context-for-wireless, pp. context-sensitive energy-efficient wireless data transfer", Proceedings of the 5th international conference on Mobile systems, applications and services, ACM, (2007), pp. 165-178.

[11] Z. A. Ying, Y. Bin, J. C. Qing and M. Qiang, "Location-Based Services: Architecture and Progress", Chinese Journal of Computers, vol. 34, no. 7, (2011), pp. 1155-1171.

[12] L. H. Feng, "Energy-efficient localization strategy for smartphones", Dalian University of Technology, (2011).

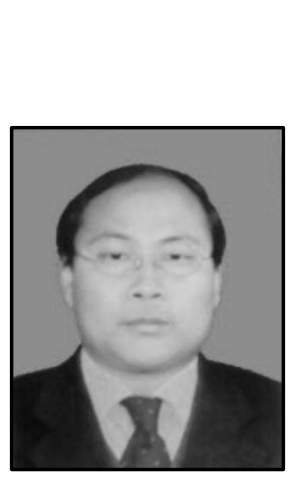

\section{Authors}

Yin Baoquan. He is a $\mathrm{Ph}$. D. candidate, a lecture at Yantai Academy of China Agricultural University. His research interests mainly involve agricultural information acquisition, prediction and early-warning technology, internet of things, etc.

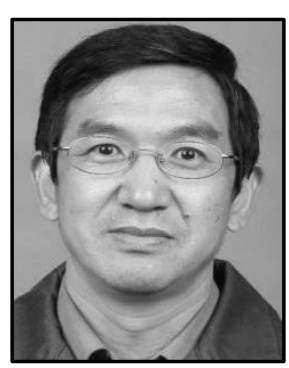

Sun Ruizhi. He is a professor, Ph.D. supervisor, Dean of Computer Science Department at China Agricultural University. He received the Ph.D. from Tsinghua University. His research interests mainly involve computer network, agricultural information acquisition technology, cooperative work and data flow, etc. 


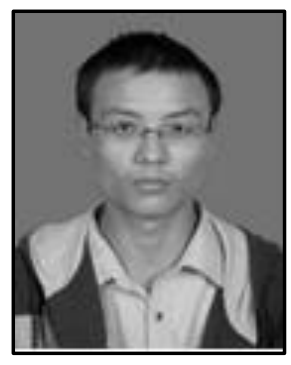

Yang HongJun. He is a postgraduate of China Agricultural University, majors in Computer Science and Technology. His research interests mainly involve Wireless Sensor Networks and Data Mining. 